\title{
Combined GC- and UHPLC-HR-MS Based Metabolomics to Analyze Durable Anti- fungal Resistance Processes in Cereals
}

\author{
Rahel Buchersa, Daniel Veyel ${ }^{b}$, Lothar Willmitzer ${ }^{b}$, Simon Krattinger $^{c}$, Beat Keller , \\ and Laurent Bigler ${ }^{\star a}$
}

§SCS-Metrohm Award for best oral presentation in Analytical Sciences

\begin{abstract}
Introduction of durable resistance genes in crops is an important strategy to prevent yield loss caused by pathogens. The durable multi-pathogen resistance gene $L r 34$ originating from wheat is widely used in breeding, and is functionally transferable to barley and rice. The molecular resistance mechanism of Lr34, encoding for an adenosine triphosphate-binding cassette transporter, is not known yet. To understand the molecular function and the defense response of durable disease resistance in cereals, the metabolic response of $L r 34$ was investigated in, except for the Lr34 gene, genetically identical lines of barley, rice and wheat. A broad range of compounds including primary, secondary and lipophilic metabolites were analyzed by a combination of gas (GC) and liquid chromatography (LC) mass spectrometry (MS) based methods. Data from metabolomics correlated well with transcriptomics data for plant defense responses such as the formation of anti-fungal hordatines or the components of the glyoxylate cycle. Induction of the glyoxylate cycle found in transgenic Lr34 rice grown in the greenhouse was confirmed in field-grown natural $L r 34$ wheat. Constitutively active plant defense responses were observed in the different cereals.
\end{abstract}

Keywords: Lr34/Yr18/Sr57/Pm38 · Mass spectrometry · Plant defense · Plant metabolomics

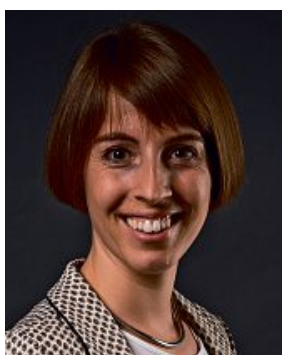

of her class. During her PhD (2012-2016), Rahel worked on plant metabolomics projects, received a SNSF Doc.Mobility grant and visited the Max Planck Institute for Molecular Plant Physiology.

\section{Introduction}

Rahel Bucher finished her apprenticeship as Textile Laboratory Assistant in 2002 and was educated as Chemist FH at the Zurich University of Applied Sciences (2003-2006). She gained industrial experience during an internship in Shanghai and working in a CRO in Switzerland (2007-2010). In 2011, she completed her Master's degree in Life Sciences at the University of Applied Sciences of Northwestern Switzerland top

${ }^{\star}$ Correspondence: Dr. L. Bigler ${ }^{a}$

E-mail: laurent.bigler@chem.uzh.ch

aDepartment of Chemistry

University of Zurich

Winterthurerstrasse 190, CH-8057 Zurich

${ }^{\mathrm{b} M a x}$ Planck Institute of Molecular Plant Physiology

Am Mühlenberg 1, DE-14476 Potsdam-Golm

'Department of Plant and Microbial Biology

University of Zurich

Zollikerstrasse 107, CH-8008 Zurich showed Lr34 resistance at seedling stage. Therefore, transgenic barley and rice plants offered a valuable tool to study the molecular differences in pathogen resistance caused by $L r 34$. The $L r 34$ resistance gene activity is associated with a senescencelike leaf-tip necrosis phenotype. [2]

In order to use plant defense against pathogens, it is crucial to understand biotic stress responses. ${ }^{[6]}$ Metabolites play vital roles in plant-pathogen interactions, and reflect the molecular phenotype. Metabolomics, with its challenging aim to identify the entire metabolite content of an organism or tissue, is a valuable tool to better understand biological processes and mechanisms, e.g. in plant biotic interactions. ${ }^{[7]}$ The workflow generally involves the analysis of the metabolic profiles, the identification of statistical differences in metabolites that are up- or downregulated upon treatment, and next the structure identification of the metabolites. Considerable challenges are the high chemical diversity associated with contrasting physicochemical properties, the wide dynamic range of metabolites, and the rapid biotransformations. ${ }^{[6]}$ They are addressed by combining multiple partially complementary analytical approaches. Metabolite identification still poses a major bottleneck in metabolomics, given the high structural diversity, the limited availability of reference com- 
pounds and the restricted phyto-metabolites in mass spectral databases.

In order to gain a deeper insight into the metabolic pathways and responses related to $\operatorname{Lr34}$, metabolomic profiling of $\operatorname{Lr} 34$ was performed as part of a multi-disciplinary approach to unravel the molecular function of durable disease resistance in cereals. Here, we present a comprehensive characterization of metabolic differences caused by Lr34 in the cereal species barley, rice and wheat. In order to find robust differences, transgenic barley and rice were grown under three well-defined conditions including hydroponic solution, mockinfection without pathogen, and infection with fungal pathogens. Finally, metabolic profiles of field-grown Lr34 wheat were analyzed to evaluate $\operatorname{Lr} 34$ function under agricultural conditions. A combination of UHPLC- and GC-MS based technologies were applied to cover a broad variety of plant metabolites including essential primary metabolites, lipids and non-essential secondary metabolites.

\section{Experimental Procedures}

Barley (Hordeum vulgare cv Golden Promise) and rice (Oryza sativa japonica cv Nipponbare) seedlings were grown under three conditions; hydroponics, mock-infected without pathogen and pathogen infected with barley leaf rust (Puccinia graminis sp hordei) or rice blast (Magnaporthe grisea), respectively. Barley and rice genotypes with low and high expression levels of Lr34res were investigated, resulting in low and high number of transcripts and respective transporter proteins. As controls, sister lines with identical genetic background lacking Lr34res were used. Flag leaves of mature fieldgrown winter wheat (Triticum aestivum $\mathrm{L}$. cv Arina) of both non-infected and wheat leaf rust (Puccinia triticina) infected plants were investigated. For all samples, whole leaves were harvested and homogenized. In order to enlarge the metabolic coverage, the plant powder was extracted by a mixture of methanol, methyl-tert-butylether (MTBE) and water, allowing the simultaneous extraction of primary and secondary metabolites as well as lipids from the same samples. ${ }^{[8]}$ Lipids were analyzed in the apolar organic fraction, ${ }^{[9]}$ whereas secondary metabolites were detected by LC-MS and primary metabolites by GC-MS ${ }^{[10]}$ in separate aliquots of the polar phase. MS data were preprocessed with TagFinder ${ }^{[11]}$ or Genedata Refiner MS (Genedata, http:// www.genedata.com) to result in two-dimensional matrices comprising metabolite identities/ feature information and intensities of all replicate samples. To account for variances that arose from sample prepara- tion and metabolite analysis, data were normalized to the intensity of the internal standards and to fresh weight of the plant material, filtered with feature intensities in blank samples, occurrence, robustness and abundance. For principle component analysis (PCA), data were $\log 2$ transformed. The univariate statistical analysis was performed by analysis of variance (ANOVA) including multiple testing corrections and further evaluation by a post-hoc Tukey's test. The significantly different metabolites with $p$-values below 0.05 were considered for comparison between independent experiments.

Secondary metabolite identification by UHPLC-HR-tandem MS (MS/MS) was performed on a QExactive Orbitrap (Thermo Fischer Scientific, Waltham, MA, US) based on calculated molecular formula, MS/MS spectra comparison to databases and literature references. Detailed experimental procedures are documented in ref. [12].

\section{Results and Discussion}

To gain a deeper insight into the metabolites and metabolic pathways related to $L r 34$ resistance, a comprehensive metabolomic profiling of barley, rice and wheat was performed. In order to find and characterize significant differences caused by the resistance gene, all experiments were designed to compare the metabolomic profiles of Lr34 plants with control plants. Known primary metabolites and lipids were assigned based on MS spectra from libraries ${ }^{[8,13]}$ and used to evaluate differences between control and Lr34 plants. For secondary metabolites, preprocessed signals from UHPLC-HR-MS, so-called features characterized by mass-to-charge ratio $(\mathrm{m} / \mathrm{z})$, retention time and intensity, were evaluated by statistical analysis to find features that significantly increased or decreased between control and Lr34 plants and were subsequently investigated for metabolite identification.

\subsection{Primary Metabolites and Lipids}

In extracts of transgenic barley and rice, eighty-four primary metabolites including amino acid derivatives, sugars, polyols and organic acids as well as 156 lipids including phospho-, sphingo-, galactolipids and triacylglycerols were found. Data from metabolic profiling were explored by multivariate analysis, namely principal component analysis (PCA). Two representative PCA score plots of primary metabolites found in mock-infected barley and rice are shown in Fig. 1. The strongest group separation was obtained for high-expressing barley (orange triangles), which was clearly separated from the highexpressing control group (orange circles) and the low-expressing groups (blue).

Significantly different metabolites between control and Lr34 plants were evaluated by univariate analysis (ANOVA), and differences that were found in replicate experiments were compared between all three growth conditions (Fig. 2). The resulting overlapping metabolites were considered as robust metabolic changes. Subsequent results were restricted to high Lr34-expressing barley, as no robust differences were observed in low expression lines or in rice.

Nine primary metabolites and sixteen lipids were changed in barley plants with high Lr34 expression levels grown under all three conditions. Amongst the robust metabolic differences, decreased dehydroascorbate concentration was related to plant defense regulation in terms of reactive oxygen species scavenging. Fructose and glucose were induced to higher levels in Lr34 barley, hinting towards reallocation of energy or association with senescence. ${ }^{[14]}$ Increased concentrations

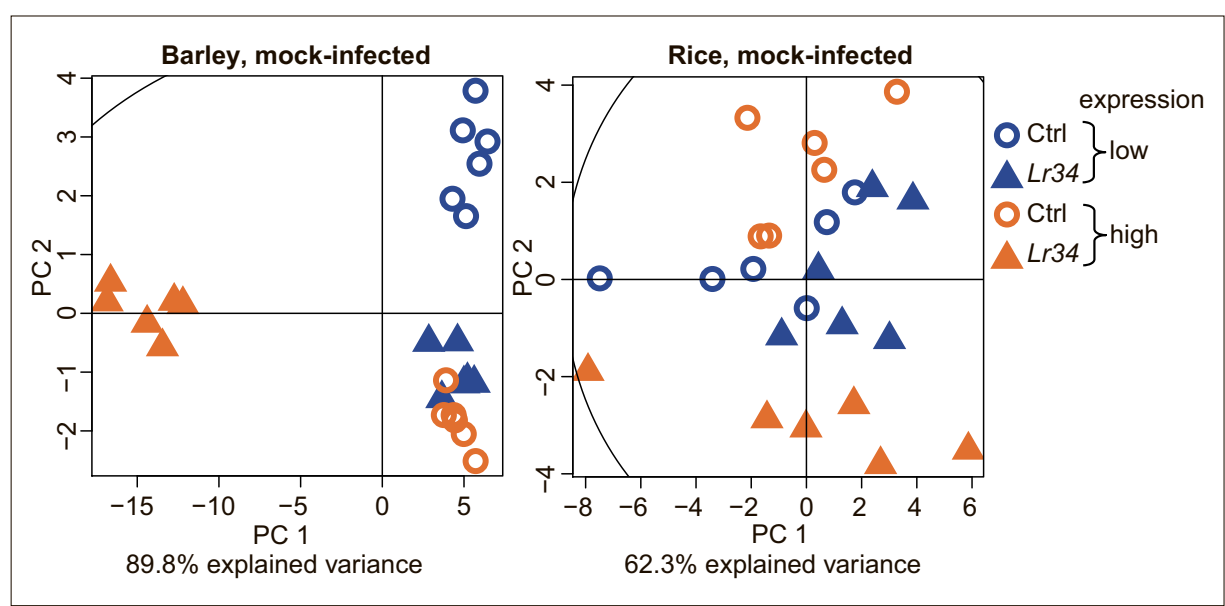

Fig. 1. Representative PCA score plots of identified primary metabolites in barley and rice grown under mock-infected conditions showing the first and second principal components (PC1, PC2). Largest group separation observed for high Lr34-expressing barley (left plot, orange triangles). 


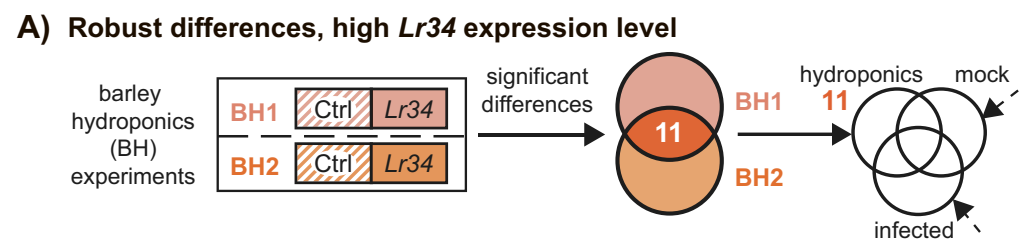

B) Primary metabolites

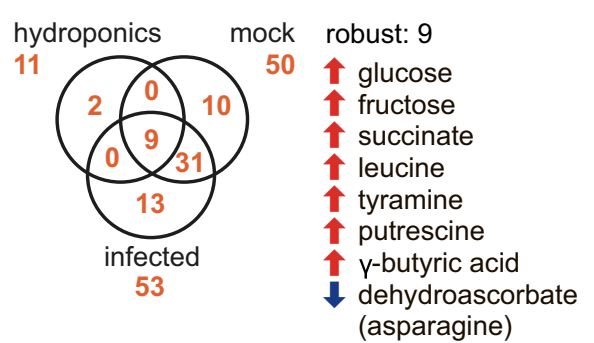

Lipids

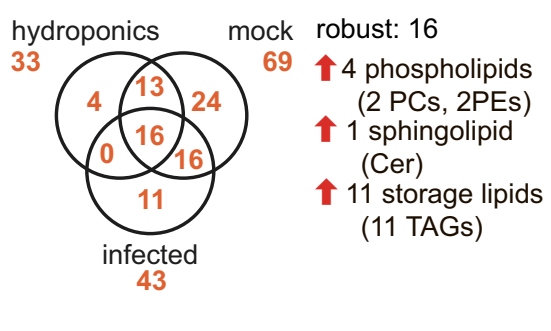

Secondary metabolite features LC-(-)-ESI-MS

\section{Secondary metabolite features LC-(+)-ESI-MS}
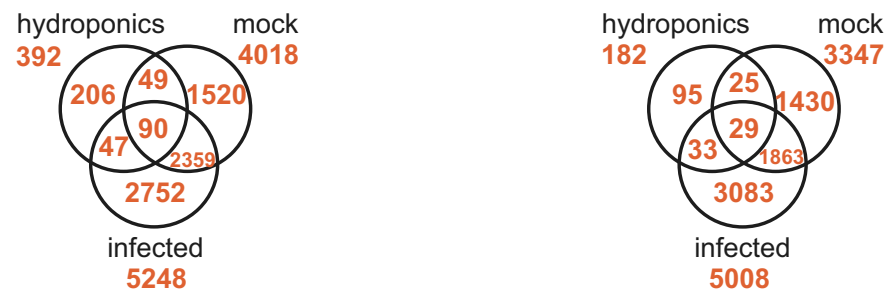

Fig. 2. Evaluation of robust significant differences in high Lr34-expressing barley; A) Significant differences between control and Lr34 were evaluated by statistical analysis, and robust differences of both replicate experiments were considered for comparison of growth conditions.

B) Robust significant differences of different metabolite classes.

of putrescine and the polyamine degradation product $\gamma$-butyric acid might be involved in plant stress tolerance. ${ }^{[15]}$ The sixteen commonly changed lipids involved each two phosphatidyl cholins (PCs) and phosphatidyl ethanolamines (PEs), one ceramide (Cer) and eleven triacylglycerols (TAGs). TAGs are formed as intermediates upon membrane breakdown during stress or senescence, and elevated sphingolipids such as ceramides regulate cell death and pathogen-induced hypersensitive responses. ${ }^{[16]}$ The observed lipid differences consequently indicate connections with plant defense responses and with the strong leaftip necrosis phenotype in barley.

Correlation of metabolomics and transcriptomics data were obtained for the glyoxylate cycle, a pathway that is induced in general stress response in rice (Fig. $3)$. Isocitrate concentrations were down-, and succinate concentrations upregulated in high-expressing transgenic Lr34 rice grown under hydroponic conditions, and also gene expression of isocitrate lyase, an enzyme in the glyoxylate cycle, was increased in Lr34 rice. ${ }^{[4]}$ The only robust changes of primary metabolites found in non-infected and infected wheat plants grown in the field were isocitrate and succinate that were increased and decreased, respectively, and a tendency of increase was also reported for gene expression of isocitrate lyase in Lr34 wheat.[17] Thus, the findings from the transgenic modelplants rice were confirmed in natural, field-grown wheat. In contrast, transgenic Lr34 barley showed a different response and isocitrate lyase gene expression was downregulated.[18]

\subsection{Secondary Metabolites}

Using the same strategy described above, a higher number of robustly significantly changed secondary metabolite features was found for high Lr34-expressing barley grown under the different conditions. Based on HR-MS and in-source fragments, a LC-(-)-electrospray ionization (ESI)-MS feature at $m / z \quad 315.0721$ and retention time $3.65 \mathrm{~min}$ was putatively assigned as gentisic acid $O$-glucoside, a glycosylated oxidation product of the plant hormone salicylic acid. A series of decreased features in high Lr34-expressing barley and rice grown under mock- and pathogen infected conditions were annotated as $C$-glycosylated flavones based on flavones previously described in literature. An example is shown in Fig. 4 for a LC-(-)-ESI-MS feature at $m / z 771.1992$ and retention time $5.21 \mathrm{~min}$. The MS/MS spectrum showed high agreement with fragments found for isoorientin-7-2"-di-O - glucoside $\left(\mathrm{C}_{23} \mathrm{H}_{38} \mathrm{O}_{20}\right)$ in barley leaves. [19] Flavonoid concentrations are known to fluctuate in response to plant stress, ${ }^{[14]}$ or the observed metabolites might undergo an unknown biotransformation. Genes of the phenylalanine pathway and flavonoid biosynthesis were induced in Lr34 barley, but the identified flavones were decreased.

Another group of barley-specific antifungal secondary metabolites called hordatines were increased in previously investigated Lr34 barley plants without pathogen infection, correlating to the increased gene expression of agmatine-coumaroyl transferase ${ }^{[18]}$ which is a key enzyme in hordatine biosynthesis. Besides the increase of hordatines, several defense responses were active in transgenic Lr34 barley even in the absence of any infection. ${ }^{[18]}$ The constitutive defense compounds being constantly synthesized by the plant suggest that Lr34 contributes to constitutive, basal plant resistance in barley and might explain the negative impact of Lr34 on the vigor of barley plants as trade-off in terms of biomass.

It is noteworthy that the majority of differentially regulated secondary metabolite features could not be identified. Plant secondary metabolites have a large structural diversity, the MS/MS spectra are difficult to reproduce and interpret, and only a few are available as reference compounds. ${ }^{[6]}$

\section{Conclusions}

The extensive metabolomic investigation of Lr34 in barley, rice and wheat characterized a variety of metabolites contributing to $L r 34$ resistance and thus helped to understand durable anti-fungal resistance processes in cereals. This study shows successful examples of integrating metabolomics and transcriptomics data, e.g. for the glyoxylate cycle or for hordatine phytoalexins. Overall, a strong, constitutive re-programming of metabolism by Lr34 in transgenic barley and rice was observed. In wheat, the results from the glyoxylate cycle were confirmed under most natural field-conditions. Multiple plant defense responses were constitutively active in Lr34 resistant plants, but the substrate of the LR34 transporter could not be identified.

\section{Acknowledgements}

This work was supported by Swiss National Science Foundation Doc.Mobility grant 158657. We thank Rainer Böni, Justine Sucher and Jyoti Singla for plant growth, Änne Michaelis and Gudrun Wolther for their excellent technical support, Dirk Steinhauser for the design of experiment and Alexander Erban and Asdrubal Burgos for their help with primary metabolite and lipid identification.

Received: January 19, 2017 
[1] S. G. Krattinger, E. S. Lagudah, T. Wicker, J. M. Risk, A. R. Ashton, L. L. Selter, T. Matsumoto, B. Keller, Plant J. 2011, 65, 392.

[2] S. G. Krattinger, E. S. Lagudah, W. Spielmeyer, R. P. Singh, J. Huerta-Espino, H. McFadden, E. Bossolini, L. L. Selter, B. Keller, Science 2009. 323,1360

[3] J. M. Risk, L. L. Selter, H. Chauhan, S. G. Krattinger, J. Kumlehn, G. Hensel, L. A. Viccars, T. M. Richardson, G. Buesing, A. Troller, E. S. Lagudah, B. Keller, Plant Biotechnol. J. 2013 11,847 .

[4] S. G. Krattinger, J. Sucher, L. L. Selter, H Chauhan, B. Zhou, M. Tang, N. M. Upadhyaya, D. Mieulet, E. Guiderdoni, D. Weidenbach, U. Schaffrath, E. S. Lagudah, B. Keller, Plant Biotechnol. J. 2015, 14, 1261.

[5] J. Sucher, R. Boni, P. Yang, P. Rogowsky, H. Buchner, C. Kastner, J. Kumlehn, S. G Krattinger, B. Keller, Plant Biotechnol. J. 2016, DOI: $10.1111 /$ pbi.12647.

[6] H. Tenenboim, Y. Brotman, Trends Plant Sci. 2016, 21, 781 .
[7] L. W. Sumner, Z. Lei, B. J. Nikolau, K. Saito, Nat. Prod. Rep. 2015, 32, 212 .

[8] P. Giavalisco, Y. Li, A. Matthes, A. Eckhardt, H.M. Hubberten, H. Hesse, S. Segu, J. Hummel, K. Kohl, L. Willmitzer, Plant J. 2011, 68, 364.

[9] B. D. Pant, A. Burgos, P. Pant, A. CuadrosInostroza, L. Willmitzer, W. R. Scheible, J. Exp. Bot. 2015, 66, 1907.

[10] J. Lisec, N. Schauer, J. Kopka, L. Willmitzer, A. R. Fernie, Nat. Protoc. 2006, 1, 387.

[11] A. Luedemann, K. Strassburg, A. Erban, J. Kopka, Bioinformatics 2008, 24, 732

[12] R. Bucher, Ph.D. Thesis, University of Zurich, 'Mass-spectrometry Based Metabolomic Approaches to Characterize Plant Pathogen Resistance in Cereals and the Flavonols Modifying Development in Arabidosis thaliana', Zurich, 2017.

[13] a) J. Kopka, N. Schauer, S. Krueger, C. Birkemeyer, B. Usadel, E. Bergmuller, P. Dormann, W. Weckwerth, Y. Gibon, M. Stitt, L. Willmitzer, A. R. Fernie, D. Steinhauser,
Bioinformatics 2005, 21, 1635; b) A. Burgos, J. Szymanski, B. Seiwert, T. Degenkolbe, M. A. Hannah, P. Giavalisco, L. Willmitzer, Plant J. 2011, 66, 656

[14] M. Watanabe, S. Balazadeh, T. Tohge, A. Erban, P. Giavalisco, J. Kopka, B. Mueller-Roeber, A R. Fernie, R. Hoefgen, Plant Physiol. 2013, 162, 1290.

[15] P. T. Do, T. Degenkolbe, A. Erban, A. G. Heyer, J. Kopka, K. I. Kohl, D. K. Hincha, E. Zuther, PLoS One 2013, 8, e60325.

[16] Y. Okazaki, K. Saito, Plant J. 2014, 79, 584.

[17] S. H. Hulbert, J. Bai, J. P. Fellers, M. G Pacheco, R. L. Bowden, Phytopathology 2007 97, 1083.

[18] H. Chauhan, R. Boni, R. Bucher, B. Kuhn, G. Buchmann, J. Sucher, L. L. Selter, G. Hensel, J. Kumlehn, L. Bigler, G. Glauser, T. Wicker, S. G. Krattinger, B. Keller, Plant J. 2015, 84, 202.

[19] A. Piasecka, A. Sawikowska, P. Krajewski, P. Kachlicki, J. Mass Spectrom. 2015, 50, 513.

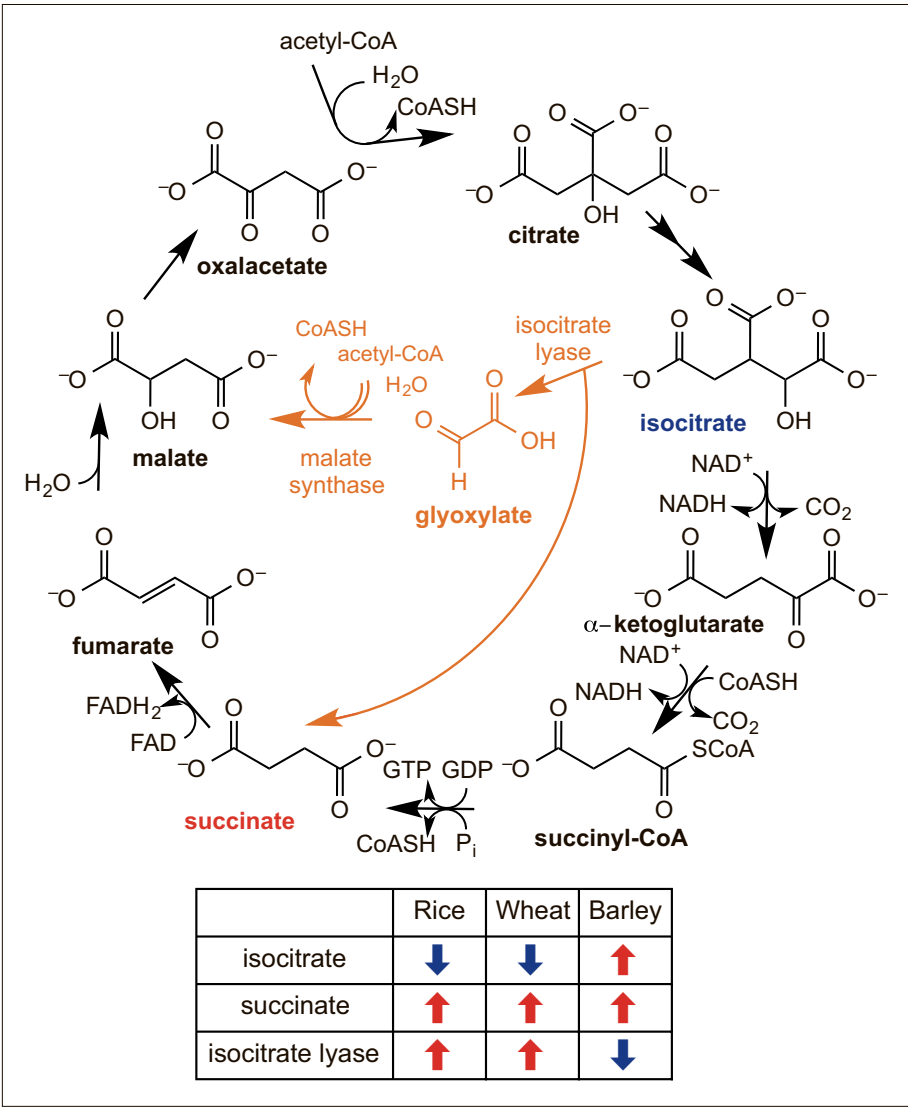

Fig. 3. Pathway interpretation of the glyoxylate cycle (orange) and citric acid cycle (black). In Lr34 plants of rice (hydroponic growth condition, high expression) and wheat (non-infected and infected), the metabolite concentrations of isocitrate were down- and succinate upregulated. The mRNA of isocitrate lyase was increased in Lr34 containing plants of rice ${ }^{[4]}$ and a tendency of increase was also reported for wheat. ${ }^{[17]}$ In barley, the metabolic response was different and isocitrate lyase gene expression downregulated. ${ }^{[18]}$

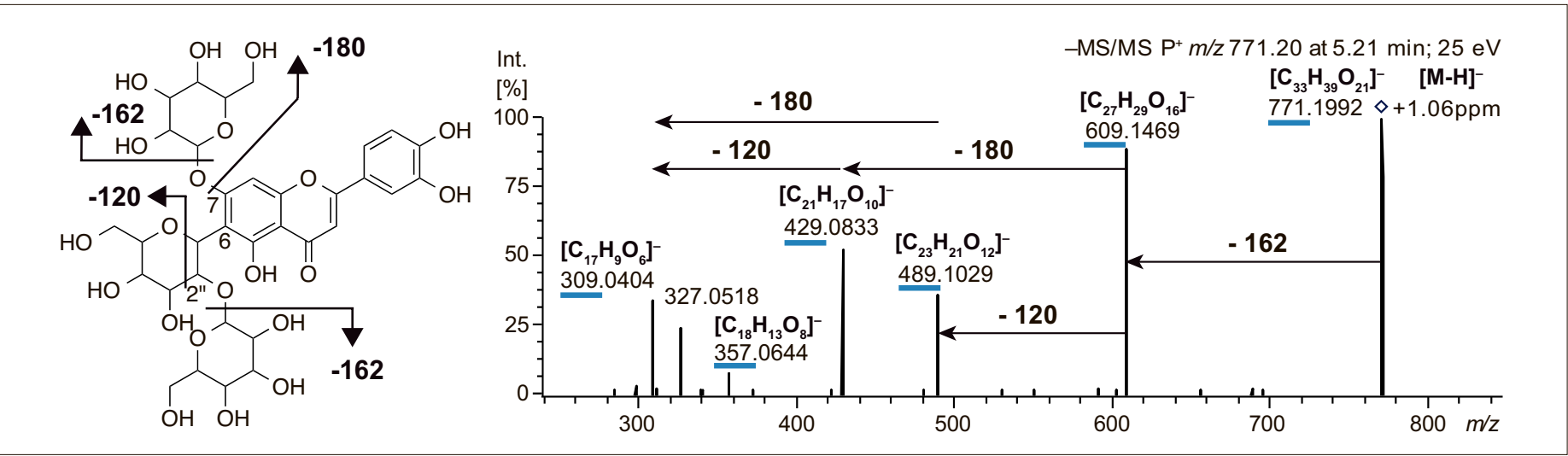

Fig. 4. LC-(-)-ESI-MS/MS of a C-glycosylated flavone [M-H] $\mathrm{m} / \mathrm{z} 771.1992$ at 5.21 min putatively assigned as isoorientin-7-2"-di-O-glucoside; fragments reported in literature are underlined. ${ }^{[19]}$ 